\title{
Regimes of adhesive wear in dry contact: Conditions of realization and determining parameters
}

Cite as: AIP Conference Proceedings 2167, 020075 (2019); https://doi.org/10.1063/1.5131942 Published Online: 19 November 2019

I. V. Dudkin, E. V. Shilko, V. L. Popov, and A. V. Dimaki

\section{ARTICLES YOU MAY BE INTERESTED IN}

Finite-element analysis of the mechanical characteristics of materials manufactured by electron-beam additive technology with metal wire

AIP Conference Proceedings 2167, 020074 (2019); https://doi.org/10.1063/1.5131941

Adhesive contribution to friction

AIP Conference Proceedings 2167, 020286 (2019); https://doi.org/10.1063/1.5132153

Influence of axial force on the pure titanium surface relief during friction stir processing

AIP Conference Proceedings 2167, 020077 (2019); https://doi.org/10.1063/1.5131944

\section{Lock-in Amplifiers up to $600 \mathrm{MHz}$

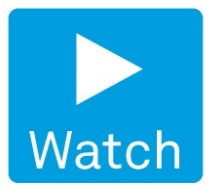

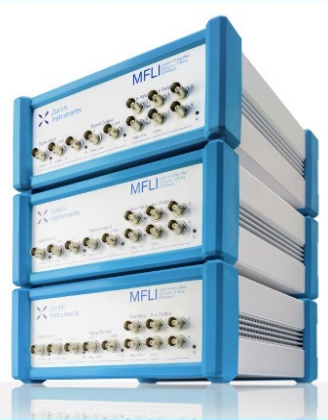




\title{
Regimes of Adhesive Wear in Dry Contact: Conditions of Realization and Determining Parameters
}

\author{
I. V. Dudkin ${ }^{1, a)}$, E. V. Shilko ${ }^{1,2, b)}$, V. L. Popov ${ }^{2,3, c)}$, and A. V. Dimaki ${ }^{1, d)}$ \\ ${ }^{1}$ Institute of Strength Physics and Materials Science SB RAS, Tomsk, 634055 Russia \\ ${ }^{2}$ National Research Tomsk State University, Tomsk, 634050 Russia \\ ${ }^{3}$ Technical University of Berlin, Berlin, Germany \\ a) Corresponding author: workdudkin@gmail.com \\ b) shilko@ispms.tsc.ru

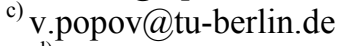 \\ d) dav@ispms.tsc.ru
}

\begin{abstract}
The paper reviews tangential adhesive contact of single asperities of elastic-plastic body surfaces. With the help of discrete element method we carried out a parametric study of influence of the parameters of adhesive interaction of the surfaces on the regime of asperity wear. It was established that with the adhesion work increase, the wear regime evolves from the mutual slippage of asperities to their attrition or brittle fracture, therewith the regime change has threshold nature. We obtained an empirical sigmoidal dependence of the threshold value of adhesion strength separating the slippage and attrition regimes on the strain-hardening coefficient of the material The results of the research make an important contribution to understanding the mechanisms that determine the development and intensity of wear in dry contact with adhesion.
\end{abstract}

\section{INTRODUCTION}

Material surfaces wear in dry friction occurs on contact patches which have a complex asperity profile $[1,2]$. The dynamics and intensity of wear are determined by the cumulative action of mechanisms that are implemented on different temporal and spatial scales. One of the key mechanisms of wear is the adhesion interaction of materials in the asperity contact patches $[1,3]$, which stimulates strain localization in the volume of asperities, their abrasion, the separation of asperities from the surface and other manifestations of wear [4]. In spite of the considerable amount of works devoted to different aspects of adhesive wear the most important macroscopic laws of wear have fully empirical character so far [5]. This determines the relevance of theoretical studies aimed at identifying the general regularities of this process and their connection with the surface asperities parameters and mechanical characteristics of materials.

Due to complexity and multifactority of the wear process, the possibilities of obtaining reliable analytical evaluations are extremely limited. One of the few examples of such evaluation is the well-known criterion of Rabinowicz [6], which links the type of elementary act of wear (asperity wear) with the specific work of adhesion value and the characteristic size of the asperity. A promising approach to the study of wear regularities is computer simulation. In particular, the results of numerical studies show that the dependence of the asperity wear intensity on the material parameters can have much more complex non-linear nature in comparison with analytical predictions [7].

In recent years, great progress has been made thanks to molecular-dynamic modeling in understanding the characteristics of adhesive wear of plastic and brittle materials with nanoscale asperity [8,9]. Despite the importance of atomistic modeling $[10,11]$ for understanding nanoscale wear mechanisms, direct transfer of the obtained results to higher scales is difficult, because on these scales the values of the mechanical characteristics that determine the plasticity and strength of the material significantly depend on the characteristics of the internal structure. This justifies the need for theoretical studies of the conditions that determine implementation of various regimes of asperity wear in the micro- and mesoscale size range.

The objective of the paper is numerical study of the effect of the macroscopic mechanical material characteristics value on the adhesive asperity wear intensity. The numerical study was conducted using the discrete element method,

Proceedings of the International Conference on Advanced Materials with Hierarchical Structure for New Technologies and Reliable Structures 2019 AIP Conf. Proc. 2167, 020075-1-020075-4; https://doi.org/10.1063/1.5131942 Published by AIP Publishing. 978-0-7354-1912-4/\$30.00 
which is widely used to model the processes of materials deformation and fracture, as well as the contact interaction of surfaces. We consider such characteristics as adhesion force of interacting asperities and the local coefficient of surfaces friction, as well as the yield point and strain-hardening coefficient of the surface layer of the material. Let us note that the identification of conditions for the specific regimes of asperity wear implementation in terms of these parameters is the foundation for reliable macroscopic models and adhesive wear criteria description.

\section{MODEL DESCRIPTION}

We used one of the implementations of the discrete element method, namely the method of movable cellular automata [12, 13]. Discrete elements were assumed as deformable and possessing isotropic elastic-plastic rheological properties. Elastic interaction of elements is described with the use of Hooke's law. Plastic deformation of elements is described on the basis of von Mises criterion of plasticity using the adapted Wilkin's algorithm. Details of numerical implementation are given in $[12,13]$.

Initially, the pairs of elements that make up the surface asperity are assumed to be chemically bound. Local fracture is modeled by breaking a chemical bond in a pair. The condition for breaking the bond is the Mises fracture criterion $\sigma_{\text {eq }}=\sigma_{c}$, where $\sigma_{\text {eq }}$ is the value of equivalent stress on the element interaction surface, $\sigma_{c}$ is the strength of the chemical bond in a pair in tension (tensile strength of the material).

Contact interaction between unbound elements includes compression resistance in the direction normal to the plane of contact (elements repulsion) and slippage resistance in the plane of contact (dry friction model based on Amonton's law). Details of the dry friction model implementation are given in the paper [14].

The interaction of unbound elements is adhesive, namely, in the absence of mechanical contact, there is an attractive central force between the elements, which is defined as the derivative of the adhesive potential. The paper uses a simplified description of such a potential in the form of Dugdale: attractive force has a constant value in the entire range of the potential. In accordance with the Dugdale model, a pair of unbound and non-contacting elements interact with the constant attractive central force $F_{\text {adh }}$, if the distance between the element surfaces does not exceed $l_{\text {adh }}$ :

$$
F_{\text {adh }}=\sigma_{\mathrm{D}} S \text {, }
$$

where $S$ is the element contact surface area, $\sigma_{\mathrm{D}}$ is the adhesion stress. The specific value of the force $\sigma_{D}$ is a material parameter. The value of $\sigma_{\mathrm{D}}$ determines the specific adhesion work (surface energy) $A_{\mathrm{adh}}=\sigma_{\mathrm{D}} l_{\mathrm{adh}}$. In the framework of the elements contact interaction model we do not consider the possibility of their "cold welding", since such a "limiting" adhesion form can be implemented only for chemically pure surfaces.

\section{RESULTS AND DISCUSSION}

We considered the tangential contact of two of elastic-plastic asperities with a trapezoidal shape and identical dimensions (the sample geometry and asperities dimensions are similar to those considered in the previous work [15]). Here, the tangential contact implies asperities interaction under the condition of their relative tangential motion. The asperities overlap value was about $5 \%$ of their height. Specified physical and mechanical properties of the contacting bodies model material are close to the typical properties of rail steel. The Dugdale's adhesive stress was a variable parameter. The adhesive potential length in all calculations was set equal to $l_{\text {adh }}=1 \mathrm{~nm}$ (the surfaces of discrete elements were assumed to be atomically smooth).

A single act of asperities interaction was modeled. The character of asperities fracture and wear at different adhesion stress values $\sigma_{D}$ was analyzed. The simulation results revealed two characteristic wear regimes, hereinafter referred to as "low-intensity wear" and "high-intensity wear".

The "low-intensity wear" regime $[2,7]$ is characterized by plastic strain accumulation in the surface layers of the contacting asperities and the formation of individual damages that are not accompanied by asperities fragmentation. Despite the fact that repeated asperities slippage may eventually lead to their fracture, as a result of gradual damage accumulation, the effective wear coefficient is rather small in the regime. This wear regime is implemented at relatively small adhesion stresses, not exceeding a certain threshold value $\sigma_{\mathrm{D}}^{*}$.

In the "high-intensity wear" regime [6], even the one-time asperities contact leads to the formation of wear particles, asperities fracture or their complete separation from the base. The wear coefficient in this interaction regime is many times higher than in the previous case. This regime is implemented when $\sigma_{\mathrm{D}}>\sigma_{\mathrm{D}}^{*}$.

The presence of the adhesion stress threshold value can be defined by the following physical explanation. Elastic strain energy accumulates in the asperities volume during the process of their contact interaction. It can be transformed (dissipated) in several ways. 
Firstly, elastic strain energy is expended on the generation and evolution of crystal lattice defects, which provide the macroscopic plasticity of the material while maintaining its continuity. Here, an important characteristic is the elastic strain energy dissipation power, which is particularly characterized by the material strain-hardening coefficient value $H$. Secondly, elastic strain energy is spent on the local asperities fracture. Important characteristics that determine the condition for implementation of this phenomenon are the strength value $\sigma_{c}$ and the critical (corresponding to fracture) value of the material elastic strain energy density $A_{\text {elast }}^{\text {crit }}=\sigma_{\mathrm{c}}^{2} / 2 E$, where $E$ is the Young's modulus of the material. Fracture occurs when the possibilities of the first dissipation path (plastic deformation) are exhausted, and the local stresses value reaches the critical point. The involvement of this dissipation path makes the implementation of the high-intensity regime on principle possible. In addition, elastic strain energy dissipates in the adhesive interaction of the areas of the original and newly formed (as a result of local destruction) surfaces located at a distance of less than $l_{\text {adh }}$ from each other. The attraction of surface areas prevents asperities slippage, so it provides effective hardening of the contact surface. The surfaces adhesion makes it possible to separate fragments of these layers (or layers as a whole) from asperity and to stick them to the opposite surface. These processes are implemented at intensive wear.

The intensive wear regime is much more energy consuming in comparison with the "non-wear" slippage regime, in which the asperities contact surfaces undergo plastic deformations. Therefore, the implementation of the intensive wear regime with tangential contact becomes possible if the adhesive surfaces interaction ensures that they reach a certain minimum (threshold) value of elastic strain energy $A_{\text {elast }}^{*}=\sigma_{\mathrm{D}}^{2} / 2 E$. Since the necessary condition for intensive asperities wear is the occurrence of damage and cracks in their volume, the threshold value $A_{\text {elast }}^{*}$ is directly determined by the value $A_{\text {elast }}^{\text {crit }}$. This corresponds to the threshold value presence of the ratio $\sigma_{D} / \sigma_{\mathrm{c}}$, when $\sigma_{\mathrm{D}} \leq \sigma_{\mathrm{D}}^{*}$ means that "non-wear" surface slippage is implemented, and the case of $\sigma_{\mathrm{D}}>\sigma_{\mathrm{D}}^{*}$ means the intensive wear regime.

Computer simulation results showed that the surface layers materials mechanical characteristics, which determine the elastic strain energy dissipation power, have a decisive influence on the interface position between the "high-intensity" and "low-intensity" wear areas (the value $\sigma_{\mathrm{D}}^{*}$ ). The key characteristic is the strain-hardening coefficient value $H$, since the energy dissipation associated with plastic deformation is involved in all the regimes of asperities interaction. The carried out parametric study showed that the dependence $\sigma_{\mathrm{D}}^{*}(H)$ has a clear-cut nonlinear logistic character and it's accurately described by a sigmoidal function (Fig. 1a). As it can be seen on Fig. 1a, in the region of small $H(H / E<0.01)$ the value $\sigma_{\mathrm{D}}^{*}$ reaches maximum magnitude (about $0.75 \sigma_{\mathrm{c}}$ in the present case) and is almost constant. As $H$ increases to $H / E \approx 0.01$, the position of the boundary between the "low-intensity" and "high-intensity" wear areas begins to decrease, i.e. "high intensity" wear occurs with weaker adhesive interaction. In the region of relatively high hardening coefficient values $(H / E>0.1)$, the intensity wear regime is implemented at sufficiently low surfaces adhesion stress values.

These results indicate the decisive role of energy dissipation associated with plastic deformation of the material in the implementation of the intensive adhesive wear regime. This is confirmed by the parametric study results of the material yield stress value effect on the wear regime. It was established that the yield stress value change over a wide range while maintaining the same elastic energy dissipation power value (the value $H$ ) practically does not affect the threshold adhesion stress value $\sigma_{\mathrm{D}}^{*}$, which separates the areas of the "low-intensity" and the "highintensity" wear regimes. Thus, the wear regime, other things being equal, is determined by the power of the energy dissipation processes carried out by the plastic deformation mechanisms, and not by the total amount of work expended on the material inelastic deformation. The features of the materials rough surfaces contact interactions are determined by the processes occurring at different scales. An effective account of the lower scales influence is traditionally carried out by introducing the friction coefficient into the contact model. The value of friction coefficient characterizes the elastic strain energy dissipation intensity on scales smaller than the characteristic scale of the contact zone. This provides the opportunity for qualitative consideration of the contact asperities surfaces as multiscale systems. The parametric study results of the dry friction coefficient (COF) effect on the asperities wear regime showed that at the same values of other mechanical characteristics an increase in COF leads to a decrease in the "threshold" adhesion stress $\sigma_{\mathrm{D}}^{*}$ (Fig. 1b). At the same time the sigmoidal nature of the ratio $\sigma_{\mathrm{D}}^{*}(H)$ remains unchanged. The indicated nature of the friction coefficient value effect has the following explanation. 

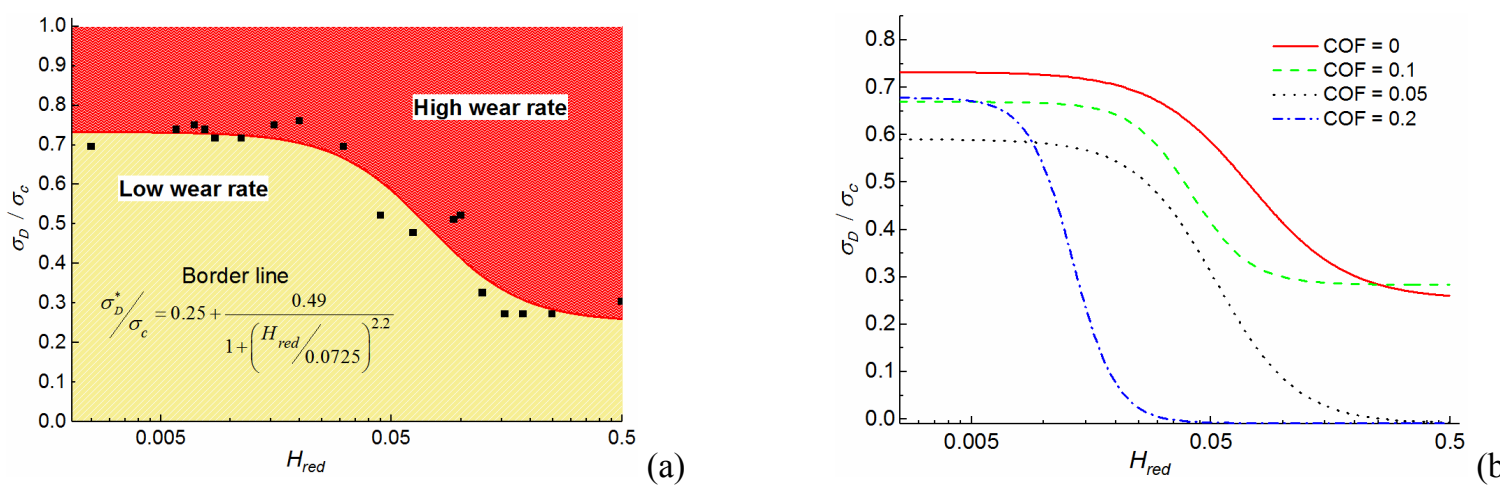

FIGURE 1. Typical dependences of the boundary position between areas of low- and high-intensity asperities wear on the normalized strain-hardening coefficient value $H_{\text {red }}=H / E$ : (a) with the dry friction coefficient value $\mathrm{COF}=0$; (b) for different

COF values. The points in (a) are the boundary positions obtained from simulation. The lines are approximating sigmoids

An increase in dry friction on asperities contact patches leads to an increase in the resistance force to the relative shear of the contacting surfaces and, consequently, to an increase in shear stresses. This contributes to the beginning of the material plastic deformation at lower values of the relative contact displacement and to an increase in the elastic energy dissipation power due to the material plastic deformation. As a result, the change of the wear regime from "low-intensity" to "high-intensity" occurs at lower values of the strain-hardening coefficient, and the "boundary sigmoid" separating the regimes shifts to the left along the abscissa (Fig. 1b).

\section{CONCLUSION}

The carried out research shows that under conditions of tangential contact of rough surfaces, the surfaces adhesion stress value is a criterion determining the implementation of the "low-intensity" or "high-intensity" wear regime of asperities. The threshold nature of change in the wear regime with a change in the surface adhesion force is a consequence of the competition in elastic strain energy dissipation through the mechanism of material plastic deformation and dissipation due to the work of adhesion. The adhesion stress threshold value $\sigma_{\mathrm{D}}^{*}$, separating these wear regimes, is a sigmoidal function of the strain-hardening coefficient of the material. The parameters of this function are non-linear functions of the local friction coefficient.

\section{ACKNOWLEDGMENTS}

The work was carried out in the framework of the Fundamental Research Program of the State Academies of Sciences for 2013-2020 (Priority direction III.23).

\section{REFERENCES}

1. A. I. Vakis, V. A. Yastrebov, J. Scheibert, et al., Tribol. Int. 125, 169-199 (2018).

2. R. Aghababaei, D. H. Warner, and J.-F. Molinari, Proc. Natl. Acad. Sci. USA 114, 7935-7940 (2017).

3. M. Ciavarella and A. Papangelo, Phys. Mesomech. 21(1), 67-75 (2018).

4. G. W. Stachowiak and A. W. Batchelor, "Adhesion and Adhesive Wear", in Engineering Tribology, edited by

G. W. Stachowiak and A. W. Batchelor (Elsevier, New York, 1993), pp. 613-635.

5. J. T. Burwell and C. D. Strand, J. Appl. Phys. 23, 18-28 (1952).

6. E. Rabinowicz, Wear 2, 4-8 (1958).

7. J. von Lautz, L. Pastewka, P. Gumbsch, and M. Moseler, Tribol. Lett. 63, 26 (2016).

8. R. Aghababaei, D. H. Warner, and J.-F. Molinari, Nat. Commun. 7, 11816 (2016).

9. T. Brink and J.-F. Molinari, Phys. Rev. Mat. 3, 053064 (2019).

10. K. P. Zolnikov, D. S. Kryzhevich, and A. V. Korchuganov, Lett. Mater. 9, 197-201 (2019).

11. A. V. Korchuganov, K. P. Zolnikov, and D. S. Kryzhevich, Mater. Lett. 252, 194-197 (2019).

12. S. Psakhie, E. Shilko, A. Smolin, et al., Frattura Integr. Strutt. 7(24), 26-59 (2013).

13. E. V. Shilko, S. G. Psakhie, S. Schmauder, V. L. Popov, S. V. Astafurov, and A. Yu. Smolin, Comp. Mater. Sci. 102, 267-285 (2015).

14. D. O. Potyondy and P. A. Cundall, Int. J. Rock. Mech. Min. Sci. 41, 1329-1364 (2004).

15. I. V. Dudkin, E. V. Shilko, and A. V. Dimaki, AIP Conf. Proc. 2051, 020069 (2018). 